\title{
Análise da Eficiência nas Taxas de Retorno e Custos de Cooperativas de Crédito Brasileiras: uma Aplicação do Método Pearls
}

Graduação em Ciências Contábeis pela Universidade Comunitária da Região de

Chapecó - UNOCHAPECÓ

Servidão Anjo da Guarda, 295-D. Efapi. Chapecó/SC. CEP: 89.809-900

E-mail: cassiabellei@unochapeco.edu.br

Vanderlei Gollo

Mestrado em Ciências Contábeis pela Universidade Regional de Blumenau - FURB Professor da Universidade Comunitária da Região de Chapecó - UNOCHAPECÓ Servidão Anjo da Guarda, 295-D. Efapi. Chapecó/SC. CEP: 89.809-900 E-mail:vande_gollo@unochapeco.edu.br

Juliana Fabris Doutorado em andamento em Administração pela Universidade do Oeste de Santa Catarina - UNOESC Professora da Universidade Comunitária da Região de Chapecó - UNOCHAPECÓ Servidão Anjo da Guarda, 295-D. Efapi. Chapecó/SC. CEP: 89.809-900 E-mail: julianafabris@unochapeco.edu.br

\begin{abstract}
Naline Tres Mestrado em andamento em Ciências Contábeis e Administração pela Universidade Comunitária da Região de Chapecó - UNOCHAPECÓ Professora da Universidade Comunitária da Região de Chapecó - UNOCHAPECÓ Servidão Anjo da Guarda, 295-D. Efapi. Chapecó/SC. CEP: 89.809-900 E-mail:nalinetres@unochapeco.edu.br
\end{abstract}

\section{RESUMO}

O estudo teve como objetivo analisar o índice de eficiência no desempenho de cooperativas de crédito a partir da aplicação PEARLS, grupo $R$ - Taxas de retorno e custos. Para tanto, utilizou-se pesquisa descritiva, documental e quantitativa a partir de uma amostra que contempla as 25 maiores cooperativas de crédito localizadas na Região Oeste do estado de Santa Catarina. Adotaram-se, para a análise dos dados, o método PEARLS, desenvolvido pelo World Council of Credit Unions. A partir do PEARLS, foram utilizados os indicadores do grupo $R$, que representam as taxas de retorno e custos, os quais foram utilizados para determinar a eficiência das cooperativas em conjunto com a metodologia TOPSIS. Os dados documentais necessários para a construção da análise foram coletados junto ao site do Banco Central do Brasil, a partir das demonstrações financeiras elaboradas de acordo com o Plano Contábil das Instituições Financeiras. Os resultados indicaram que a eficiência no desempenho das 
Análise da Eficiência nas Taxas de Retorno e Custos de Cooperativas de Crédito Brasileiras: uma

Aplicação do Método Pearls

Cassia Bellei, Vanderlei Gollo, Juliana Fabris, Naline Tres

cooperativas de crédito não está relacionado ao seu tamanho. Isso reforça a importância das informações contábeis para a análise das cooperativas de crédito, uma vez que as mais eficientes não apresentaram relação com o tamanho ou tempo de existência.

Palavras-chave: Instituição Financeira. Rentabilidade. TOPSIS.

\section{Analysis of Efficiency in Return Rates and Costs of Brazilian Credit Unions: An Application of the Pearls Method}

\section{ABSTRACT}

The objective of this study was to analyze the performance efficiency index of credit unions from the PEARLS application, group $R$ - Return rates and costs. For this, we used a descriptive, documentary and quantitative research from a sample that includes the 25 largest credit unions located in the western region of the state of Santa Catarina, Brazil. For the data analysis, the PEARLS method, developed by the World Council of Credit Unions, was adopted. From the PEARLS, we used the group R indicators, which represent the return rates and costs, which were used to determine the efficiency of the cooperatives in conjunction with the TOPSIS methodology. The documentary data necessary for the construction of the analysis were collected from the Central Bank of Brazil website, from the financial statements prepared in accordance with the Accounting Plan of the Financial Institutions. The results indicated that the efficiency in the performance of credit unions is not related to their size. This reinforces the importance of accounting information for the analysis of credit unions, since the most efficient ones were not related to size or time of existence.

Keywords: Financial Institution. Profitability. TOPSIS.

\section{INTRODUÇÃO}

A procura pelos serviços prestados pelas cooperativas de crédito é cada vez maior e deve-se, principalmente, pelas melhores taxas para operações financeiras (empréstimos e financiamentos), quando comparadas aos bancos privados. Atualmente, os governos têm estimulado as pessoas a utilizarem serviços ligados às cooperativas de crédito por meio de incentivos à disponibilização de linhas de crédito atrativas, o que está gerando grande aumento no índice de procura (Araújo \& Silva, 2011). 
Análise da Eficiência nas Taxas de Retorno e Custos de Cooperativas de Crédito Brasileiras: uma Aplicação do Método Pearls

Cassia Bellei, Vanderlei Gollo, Juliana Fabris, Naline Tres

As cooperativas de crédito são instituições financeiras caracterizadas pela prestação de serviços financeiros exclusivamente aos associados, que são donos e usuários dos serviços. Em geral, são pelo menos vinte sócios que compõem o quadro social, com capitalização de recursos e que têm direito à distribuição das sobras, proporcionalmente às operações que cada associado realiza com a cooperativa. As cooperativas de crédito são classificadas como captadoras de depósito à vista, supervisionadas pelo Banco Central do Brasil [BACEN] (Soares \& Melo, 2007).

Segundo McKillop, Glass e Ferguson (2002), a força maior do cooperativismo de crédito decorre da filosofia e objetivos básicos, pois não visa ao lucro; os direitos e deveres dos cooperados são iguais; o voto é igualitário entre os associados, independentemente da cota de participação no capital social da cooperativa; e a adesão é livre e voluntária.

Estudos anteriores identificaram que o desempenho, em um ranking global, nas cooperativas de crédito vem crescendo nos últimos anos. Para tanto, a metodologia gerenciada para controle de finanças cooperativistas foi o método PEARLS. Tal método possibilita a comparação de resultados econômicos entre as contas das demonstrações financeiras, podendo apresentar com confiabilidade seus resultados (Bressan, Braga, Bressan, \& Resende, 2011).

Indicadores contábil-financeiros inéditos, obedecendo às orientações do Método PEARLS, criado pelo World Council of Credit Unions [WOCCU], que é o Conselho Mundial das Cooperativas de Crédito, são destacados por Bressan, Bressan, Oliveira, \& Braga (2014). O modelo é utilizado em vários países e, até então, segundo Woccu (2014), não havia sido pesquisado no Brasil pela necessidade de uma adaptação ao ambiente nacional. O método PEARLS foi criado pelo Woccu no final década de 1980 , e os indicadores são adotados em 97 países distribuídos na África, Ásia, Caribe, Europa, América do Norte, América Latina e Oceania (Woccu, 2014). A aplicação do sistema PEARLS à realidade brasileira possui especificação das contas do plano Contábil das Instituições Financeiras do Sistema Financeiro Nacional (COSIF) por indicadores contábeis financeiros (Bressan et al., 2014). 
A análise dos indicadores econômico-financeiros caracteriza-se por considerar informações de vários demonstrativos contábeis, podendo identificar a evolução do desempenho econômico-financeiro da entidade, além de projetar resultados futuros (Kaplan \& Cooper, 2000; Camargos \& Barbosa, 2005).

Considerando-se a importância do desempenho econômico-financeiro na obtenção de eficiência para que as cooperativas de crédito possam atingir aos seus objetivos, voltados a atender às necessidades de seus associados, formula-se a questão de pesquisa: Qual a eficiência no desempenho das cooperativas de crédito a partir da aplicação da metodologia PEARLS, grupo R "Taxas de retorno e custos"?

A partir desse questionamento, o objetivo do estudo é analisar o índice de eficiência no desempenho de cooperativas de crédito a partir da aplicação da metodologia PEARLS, grupo R: "Taxas de retorno e custos".

Dessa forma, o artigo está assim estruturado: esta introdução, seguida da breve revisão de literatura sobre os temas cooperativismo de crédito e método PEARLS. Depois são detalhados os procedimentos metodológicos que amparam o tópico de análise e interpretação dos resultados, seguidos pelas considerações finais e referências.

\section{REVISÃO DA LITERATURA}

Este tópico aborda inicialmente a história, origem e conceitos relacionados ao cooperativismo de crédito e, na sequência, trata da aplicabilidade do método PEARLS, seus objetivos e interesse para as cooperativas de crédito, evidenciando o índice de risco "R".

\subsection{Cooperativismo de Crédito}

As primeiras cooperativas de crédito surgiram na cidade alemã de Delitzsch, tendo como precursor Franz Herman Schulze, em 1852, com a criação da cooperativa de crédito urbana, seguindo o modelo denominado Schulze-Delitzsch. Anos depois, 
foram criadas as cooperativas de crédito rural por Friedrich Wilhelm Raiffeisen, em 1862, em Anhausen, e, em 1864, em Heddesdorf, ambas na Alemanha. Mais tarde, elas assumiram o nome de Raiffeisen bank, seguindo o modelo Raiffeisen e, em 1865, na Itália, surgem as primeiras cooperativas de crédito de livre associação do modelo Luzzatti, tendo como precursor Luigi Luzzatti (Menezes \& Lajus, 2015).

O cooperativismo de crédito, segundo Lima, Silva e Lima (2013), teve início no Brasil em 28 de dezembro de 1902, no município de Nova Petrópolis/RS, baseado nos ideais do Padre Theodor Amstad, que criou a Caixa de Economia e Empréstimos Amstad, hoje conhecida como Cooperativa de Crédito de Livre Admissão de Associados Pioneira da Serra Gaúcha - Sicredi Pioneira RS, seguindo o modelo alemão Raiffeisen.

O cooperativismo tem por objetivo melhorar a vida das pessoas e das comunidades (Woccu, 2018), o desenvolvimento do ser humano e das famílias, podendo ser criado por vinte ou mais pessoas que se unem, voluntariamente, para satisfazer seus interesses financeiros, agregando menores taxas, com serviços comparados aos de um banco (Lima, Silva, \& Lima, 2013).

Em junho de 2008, o sistema cooperativo de crédito no Brasil encontrava-se estruturado com dois bancos cooperativos, sendo um múltiplo e o outro comercial, cinco confederações, uma federação, 38 cooperativas centrais e 1.423 cooperativas singulares, com 4.044 pontos de atendimento, somando mais de três milhões de associados. Dentre as singulares, 152 eram de livre admissão de associados, 74 eram de empresários, 386 eram de crédito rural e 881 eram dos demais tipos. No entanto, desde a década de 1940, constata-se que as cooperativas de crédito efetivamente estavam registradas nos órgãos oficiais do Governo (Pinheiro, 2008).

Com o decorrer dos anos e, a partir das necessidades das cooperativas de crédito, foram criados os bancos cooperativos, por exemplo: o Bansicredi, em 1995, e o Bancoob, em 1996 (Lima, Silva, \& Lima, 2013). No intuito de auxiliar na redução dos custos e na prestação dos serviços, os bancos cooperativos atuam como um instrumento para as cooperativas de crédito, administrando os recursos e 
desenvolvendo produtos e políticas de comunicação mais eficazes, a fim de melhorar a qualidade nos serviços prestados a seus cooperados. O Conselho Monetário Nacional, por meio da Resolução 3.106/2003, permitiu a constituição das cooperativas de livre admissão. Isso possibilitou o desenvolvimento do cooperativismo de crédito (Cunha, Santos, Bezerra, \& Pinto, 2010). A referida resolução foi revogada pela Resolução $3.321 / 2005$, que autoriza a constituição de cooperativas de livre admissão em regiões com até 300 mil habitantes (Cunha et al., 2010; Chaves, 2011).

As cooperativas de crédito, segundo Bressan et al. (2014), estão assumindo um grande desafio quando se trata de criar mecanismos de gestão eficazes que atendam as exigências regulatórias BACEN e que estejam em conformidade com os princípios doutrinários. Entre os desafios, destaca-se o sistema PEARLS (P-Protection; EEffective financial structure; A-Asset quality; R-Rates of return and costs; L-Liquidity e SSigns of growth), que é um mecanismo de gestão e monitoramento do desempenho financeiro para cooperativas de crédito, utilizado em diferentes países e recomendado pelo Woccu (2014). PEARLS é um acrônimo composto por um conjunto de índices e indicadores financeiros que têm por objetivo atender as particularidades das cooperativas de crédito, oferecendo uma ferramenta de gerenciamento com padronização dos índices, permitindo a comparação entre outras cooperativas (Woccu, 2014).

De acordo com Bressan et al. (2014), esse sistema funciona como um "avisoprévio" às cooperativas de crédito. Oliveira, Bressan e Bressan (2014) destacam os seguintes itens que compõem o acrônimo PEARLS: proteção, estrutura financeira efetiva, qualidade dos ativos, taxas de retorno e custos, liquidez e sinais de crescimento.

Diante da eficácia, pelo fato do PEARLS ser utilizado como método de gestão pelas cooperativas de crédito em vários países, alguns pesquisadores utilizaram este sistema como base para a realização de estudos no Brasil, adaptando-o de acordo com as necessidades das cooperativas, entre os quais se destacam: Bressan et al. (2011); Araújo e Silva (2011); Bressan et al. (2014); Oliveira, Bressan e Bressan (2014); Gollo e 
Análise da Eficiência nas Taxas de Retorno e Custos de Cooperativas de Crédito Brasileiras: uma Aplicação do Método Pearls Cassia Bellei, Vanderlei Gollo, Juliana Fabris, Naline Tres

Da Silva (2015); e Silva, Padilha e Silva (2015). Estes estudos estão voltados a identificar a insolvência em cooperativas de crédito, em analisar o desempenho das cooperativas e identificar quais indicadores são considerados relevantes para o seu desempenho.

\subsection{Método PEARLS (Taxas de Retorno e Custos) "R"}

O método PEARLS foi criado para aprimorar os efeitos de relevância nas cooperativas de crédito. Seu objetivo é indicar os maiores riscos e analisar o desempenho especificamente das cooperativas de crédito. Além dos indicadores, o sistema PEARLS pode destacar quanto uma cooperativa tem de estrutura em capital próprio e também identifica seus graus de problemas. Em sistema, o método PEARLS é conhecido praticamente por ser um "aviso prévio" capaz de gerar informações suficientes para uma melhor tomada de decisão (Bressan et al., 2011).

O sistema PEARLS é considerado por Richardson (2002) como uma ferramenta de gestão que auxilia o gestor a encontrar e prevenir futuros problemas em uma cooperativa de crédito. É capaz de monitorar o desempenho de uma cooperativa de crédito, mostrando se a cooperativa poderá sofrer inadimplência alta, insuficiência de receita ou, até mesmo, se as despesas ficarão acima do normal.

A composição do sistema PEARLS é formada por índices que estão presentes nas contas COSIF aplicadas às cooperativas de crédito. O nome PEARLS, que significa "pérolas" em português, compõe-se da seguinte forma: $P$ = Protection (proteção); $E$ = Effective financial structure (estrutura financeira efetiva); $A=$ Assets quality (qualidade dos ativos); $R=$ Rates of return and costs (taxas de retorno e custos); $L=$ Liquidity (liquidez); e, $\mathrm{S}=$ Signs of growth (sinais de crescimento), como explicam Bressan et al. (2011).

Em relação às Taxas de Retorno e Custos - Rates of Return and Costs, o sistema PEARLS segrega todos os componentes essenciais das rendas líquidas para auxiliar no gerenciamento dos rendimentos dos investimentos e na avaliação das despesas operacionais. Dessa maneira, PEARLS demonstra seu valor como uma 
Análise da Eficiência nas Taxas de Retorno e Custos de Cooperativas de Crédito Brasileiras: uma Aplicação do Método Pearls

Cassia Bellei, Vanderlei Gollo, Juliana Fabris, Naline Tres

ferramenta de gerenciamento. Diferentemente de outros sistemas que calculam rendimentos em base de ativos médios, PEARLS calcula rendimentos em base de investimentos reais ótimos (Gollo \& Da Silva, 2015). Também permite que as cooperativas de crédito sejam classificadas segundo os melhores e piores rendimentos. Comparando-se a estrutura financeira com os rendimentos, é possível determinar quão eficazmente a cooperativa de crédito pode colocar seus recursos produtivos em investimentos que produzem rendimento mais elevado.

\begin{tabular}{|c|c|}
\hline Indicador & Proxy \\
\hline R1 & Rendas de operações de crédito/ Média das operações de crédito \\
\hline R2 & Renda líquida de investimento financeiro/ Investimento financeiro médio \\
\hline R3 & Despesas de depósito a prazo/ Depósitos a prazo \\
\hline R4 & $\begin{array}{c}\text { Despesas de obrigações por empréstimos e repasses/ Média das obrigações por } \\
\text { empréstimos e repasses }\end{array}$ \\
\hline R5 & Margem bruta/ Ativo total médio \\
\hline R6 & Despesas operacionais/ Ativo total médio \\
\hline R7 & Sobras/ Ativo total médio \\
\hline R8 & Sobras/ Patrimônio líquido ajustado médio \\
\hline R9 & Resultado da intermediação financeira/ Receita operacional \\
\hline R10 & Sobras/Receita operacional \\
\hline R11 & Rendas de prestação de serviços/ Despesas administrativas \\
\hline R12 & Despesas de gestão/ Despesas administrativas \\
\hline R13 & Despesas administrativas/Ativo total médio \\
\hline
\end{tabular}

Figura 1. Indicadores das taxas de retorno e custos Fonte: Bressan et al. (2011).

Na Figura 1, podem ser observados os indicadores utilizados para análise do índice de eficiência das cooperativas de crédito. Esses indicadores representam as taxas de retorno e custos das cooperativas, a partir de suas operações, pelos quais é possível medir o desempenho dessas organizações.

\section{PROCEDIMENTOS METODOLÓGICOS}

Os procedimentos metodológicos de pesquisa em contabilidade podem ser enquadrados em três categorias: quanto aos seus objetivos, quanto aos procedimentos e quanto à abordagem do problema (Raupp \& Beuren, 2012). Assim, para atender ao 
objetivo de analisar o índice de eficiência no desempenho de cooperativas de crédito a partir da aplicação PEARLS, grupo R, Taxas de retorno e custos, desenvolveu-se uma pesquisa descritiva, documental e quantitativa.

A classificação da pesquisa quanto aos objetivos é caracterizada como descritiva, pois visa identificar, relatar e comparar as variáveis, aspectos ou comportamentos de determinadas populações (Raupp \& Beuren, 2012), ou seja, tem como principal objetivo descrever características de determinada população, utilizando técnicas padronizadas para a coleta de dados (Gil, 2019). A pesquisa é documental quanto aos procedimentos e se concentra no estudo do risco de crédito. A abordagem é quantitativa por dar maior ênfase a dados econômico-financeiros coletados a partir das demonstrações financeiras das cooperativas integrantes do estudo.

A população do estudo compreende as cooperativas de crédito do oeste catarinense, com amostra composta pelas 25 maiores cooperativas, a partir do ranking divulgado pelo Banco Central do Brasil em 31 de junho de 2017, para justificar os índices de risco de crédito das 38 cooperativas de crédito durante o período de 2015, 2016 e 2017. 
Análise da Eficiência nas Taxas de Retorno e Custos de Cooperativas de Crédito Brasileiras: uma Aplicação do Método Pearls

Cassia Bellei, Vanderlei Gollo, Juliana Fabris, Naline Tres

\begin{tabular}{|c|c|c|}
\hline COOPERATIVA & CIDADE SEDE & RANKING BACEN \\
\hline Sicoob Maxicrédito & Chapecó & $1^{0}$ \\
\hline Sicoob São Miguel SC & São Miguel D'Oeste & $2^{\circ}$ \\
\hline Sicoob Crediauc SC & Concórdia & $3^{0}$ \\
\hline Sicoob Creditaipu & Pinhalzinho & $4^{0}$ \\
\hline Sicoob Credial SC & Cunha Porã & $5^{0}$ \\
\hline Unicredi Oeste e Seara & Chapecó & $6^{0}$ \\
\hline Sicoob Videira SC & Videira & $7^{0}$ \\
\hline Sicoob Oestecredi & Palmitos & $8^{\circ}$ \\
\hline Sicoob Transcredi & Concórdia & $9^{\circ}$ \\
\hline Sicoob Creditapiranga SC/RS & Itapiranga & $10^{\circ}$ \\
\hline Sicoob Valcredi Sul & Passos Maia & $11^{\circ}$ \\
\hline Sicoob Crediplanalto SC/RS & Papanduva & $12^{\circ}$ \\
\hline Sicoob Credirio SC & Joaçaba & $13^{\circ}$ \\
\hline Sicoob Credimoc SC & Xanxerê & $14^{\circ}$ \\
\hline Sicoob Noroeste SC & São Lourenço D'Oeste & $15^{\circ}$ \\
\hline Sulcredi Crediluz & Abelardo Luz & $16^{\circ}$ \\
\hline Crediseara & Seara & $17^{\circ}$ \\
\hline Cresol Xavantina & Xavantina & $18^{\circ}$ \\
\hline Cresol Xaxim & Xaxim & $19^{\circ}$ \\
\hline Coliberte & Joaçaba & $20^{\circ}$ \\
\hline Sulcredi Ouro & Ouro & $21^{\circ}$ \\
\hline Cresol Concórdia & Concórdia & $22^{\circ}$ \\
\hline Cresol Pedra Branca & Coronel Martins & $23^{\circ}$ \\
\hline Cresol Chapecó & Chapecó & $24^{\circ}$ \\
\hline Sicoob Caçador SC & Caçador & $25^{\circ}$ \\
\hline
\end{tabular}

Figura 2. Ranking das 25 maiores cooperativas de crédito da região Oeste de Santa Catarina. Fonte: Dados da pesquisa.

De acordo com dados apresentados por Soares e Melo (2007), o Sistema de Cooperativas de Crédito do Brasil (Sicoob) congrega 46,32\% das cooperativas singulares brasileiras e é o maior entre os sistemas existentes no país, quais sejam: Sicoob, Sicredi, Unicred, Ancosol, Cooperativas Independentes e outros sistemas. O Sicoob é composto por 14 cooperativas centrais, 633 cooperativas singulares de crédito, 1.144 postos de atendimento cooperativo (PA) e conta com a participação de 1,7 milhões de associados e com a Confederação Nacional de Cooperativas de Crédito (Sicoob-Brasil). De acordo com dados de dezembro de 2008, o Sicoob possuía $\mathrm{R} \$ 9$ bilhões em operações de crédito, $R \$ 7,1$ bilhões em depósitos, $R \$ 4$ bilhões em patrimônio de referência, $R \$ 551,8$ milhões de resultado anual e $R \$ 14,8$ bilhões de ativos totais (Soares \& Melo, 2007). 
Análise da Eficiência nas Taxas de Retorno e Custos de Cooperativas de Crédito Brasileiras: uma Aplicação do Método Pearls Cassia Bellei, Vanderlei Gollo, Juliana Fabris, Naline Tres

O conjunto de cooperativas singulares que compõem a amostra do presente estudo pertence a diversos sistemas cooperativos do país. São instituições financeiras que se destinam à prestação de serviços financeiros a seus associados, de forma a garantir o acesso às ferramentas do mercado financeiro (Maia, Bressan, Lamounier, \& Braga, 2013).

Os dados documentais foram coletados junto ao BACEN (2019) a partir das demonstrações financeiras elaboradas de acordo com o Plano de Contas das Instituições do Sistema Financeiro Nacional (COSIF). A amostra é composta por 25 cooperativas de crédito localizadas na região oeste do estado de Santa Catarina, com coleta dos dados realizada no período de outubro a dezembro de 2016, para os exercícios sociais entre os anos de 2013 a 2015. Ressalta-se que, para a construção dos indicadores, foi também necessário coletar informações referentes ao período de 2012.

Após a realização da coleta dos dados, estes foram selecionados e organizados de acordo com as contas utilizadas para aplicação do cálculo dos indicadores do sistema PEARLS. A análise dos dados coletados tomou por base o estudo de Gollo e Da Silva (2015), que agregam um conjunto de indicadores do sistema PEARLS, e foi empregada uma técnica estatística por meio do método TOPSIS, para identificar o ranking das cooperativas em estudo. Nesse sentido, Bressan et al. (2011; 2014) buscaram adequar os indicadores do PEARLS à realidade das cooperativas brasileiras, a fim de fornecer aos gestores um critério de comparação ao longo do tempo e facilitar o controle e supervisão.

\section{ANÁLISE E INTERPRETAÇÃO DOS RESULTADOS}

O método TOPSIS, de acordo com Bulgurcu (2012), é uma técnica utilizada para determinar o ranking de alternativas para obter a melhor alternativa de um cenário, sendo a mais próxima da solução ideal. Em outras palavras, esse método considera os maiores scores de cada período analisado como sendo o cenário ideal $(1,000)$ e os 
Análise da Eficiência nas Taxas de Retorno e Custos de Cooperativas de Crédito Brasileiras: uma Aplicação do Método Pearls Cassia Bellei, Vanderlei Gollo, Juliana Fabris, Naline Tres

menores scores o anti-ideal $(0,000)$. Assim, o método TOPSIS leva em consideração a distância de ambos os lados para a determinação dos scores.

Com o objetivo de determinar eficiência no Rates of return and costs (taxas de retorno e custos) das cooperativas de crédito da região Oeste de Santa Catarina, após a coleta e organização dos dados, foi realizado o cálculo das variáveis dos grupos de indicadores do PEARLS, bem como aplicou-se o método TOPSIS para a formação do ranking de cada cooperativa para todos os períodos analisados.

A pesquisa, assim como o estudo de Oliveira, Bressan e Bressan (2014) reforça a relevância das informações contábeis para a construção de sistemas de avaliação e auxílio aos gestores no processo de tomada de decisões. Todas as informações que constam no sistema PEARLS para o cálculo de seus indicadores são provenientes das demonstrações contábeis divulgadas de cada cooperativa.

A partir da utilização do TOPSIS, foi gerado score por grupo de indicadores para cada uma das 24 cooperativas nos períodos-base de 2015, 2016 e 2017. A Tabela 1 foi elaborada para demonstrar a eficiência das cooperativas analisadas em cada um dos períodos da análise. 
Análise da Eficiência nas Taxas de Retorno e Custos de Cooperativas de Crédito Brasileiras: uma

Aplicação do Método Pearls

Cassia Bellei, Vanderlei Gollo, Juliana Fabris, Naline Tres

Tabela 1

Topsis - Eficiência das cooperativas de crédito por período de análise

\begin{tabular}{|c|c|c|c|c|c|c|c|}
\hline \multirow[t]{2}{*}{ Cooperativas } & \multirow{2}{*}{$\begin{array}{l}\text { Ranking } \\
\text { BACEN }\end{array}$} & \multicolumn{6}{|c|}{ TOPSIS } \\
\hline & & Posição & Score & Posição & Score & Posição & Score \\
\hline Sicoob Maxicrédito & $1 \stackrel{0}{\underline{0}}$ & 9o & 0,5750 & $4^{\circ} \stackrel{0}{0}$ & 0,5262 & $21^{\circ}$ & 0,4839 \\
\hline Sicoob São Miguel SC & $2^{0}$ & $5^{0}$ & 0,5983 & $18^{\circ}$ & 0,4844 & $1 \stackrel{0}{1}$ & 0,6067 \\
\hline Sicoob Crediauc SC & $3^{\circ}$ & $11^{\circ}$ & 0,5720 & $16^{\circ}$ & 0,4912 & $12^{0}$ & 0,5159 \\
\hline Sicoob Creditaipu & $4^{\circ}$ & $14^{\circ}$ & 0,5623 & $17^{\circ}$ & 0,4885 & $18^{\circ}$ & 0,4969 \\
\hline Sicoob Credial SC & $5^{\circ}$ & $8^{\circ}$ & 0,5815 & $10^{\circ}$ & 0,5014 & $10^{\circ}$ & 0,5301 \\
\hline Unicredi Oeste e Seara & $6^{\circ}$ & $20^{\circ}$ & 0,5522 & $1 \stackrel{0}{1}$ & 0,7522 & $8^{\circ}$ & 0,5390 \\
\hline Sicoob Videira SC & $7^{0}$ & $18^{\circ}$ & 0,5574 & $6^{0}$ & 0,5177 & $22^{\circ}$ & 0,4723 \\
\hline Sicoob Oestecredi & $8^{0}$ & $10^{\circ}$ & 0,5727 & $20^{\circ}$ & 0,4799 & $24^{\circ}$ & 0,4088 \\
\hline Sicoob Transcredi & 90 & $12^{0}$ & 0,5701 & $13^{\circ}$ & 0,4928 & $14^{\circ}$ & 0,5070 \\
\hline Sicoob Creditapiranga SC/RS & $10^{\circ}$ & $7^{0}$ & 0,5883 & $9^{0}$ & 0,5109 & $5^{\circ}$ & 0,5526 \\
\hline Sicoob Valcredi Sul & $11^{\circ}$ & $18^{\circ}$ & 0,5533 & $23^{\circ}$ & 0,4342 & $20^{\circ}$ & 0,4845 \\
\hline Sicoob Crediplanalto SC/RS & $12^{\circ}$ & $15^{\circ}$ & 0,5594 & $8^{0}$ & 0,5118 & $19^{\circ}$ & 0,4967 \\
\hline Sicoob Credirio SC & $13^{0}$ & $4^{\circ}$ & 0,6075 & $3^{\circ}$ & 0,5422 & $11^{\circ}$ & 0,5243 \\
\hline Sicoob Credimoc SC & $14^{\circ}$ & $16^{\circ}$ & 0,5585 & $22^{\circ}$ & 0,4417 & $7^{0}$ & 0,5444 \\
\hline Sicoob Noroeste SC & $15^{0}$ & $17^{\circ}$ & 0,5581 & $19^{\circ}$ & 0,4844 & $2^{0}$ & 0,6067 \\
\hline Sulcredi Crediluz & $16^{0}$ & 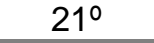 & 0,5475 & $24^{\circ}$ & 0,4021 & $17^{\circ}$ & 0,4994 \\
\hline Crediseara & $17^{\circ}$ & $1 \stackrel{0}{1}$ & 0,6425 & $7^{0}$ & 0,5161 & $13^{\circ}$ & 0,5141 \\
\hline Cresol Xavantina & $18^{\circ}$ & $2^{0}$ & 0,6222 & 5 & 0,5182 & $3^{\circ}$ & 0,5649 \\
\hline Cresol Xaxim & $19^{\circ}$ & $3^{0}$ & 0,6203 & $2^{o}$ & 0,5430 & $4^{0}$ & 0,5564 \\
\hline Sulcredi Ouro & $20^{\circ}$ & $22^{\circ}$ & 0,5473 & $14^{\circ}$ & 0,4928 & $15^{\circ}$ & 0,5070 \\
\hline Cresol Concórdia & $21^{\circ}$ & $24^{\circ}$ & 0,4994 & $15^{\circ}$ & 0,4928 & $16^{\circ}$ & 0,5070 \\
\hline Cresol Pedra Branca & $22^{\circ}$ & $23^{\circ}$ & 0,5442 & $21^{\circ}$ & 0,4499 & 9o & 0,5343 \\
\hline Cresol Chapecó & $23^{\circ}$ & $6^{\circ}$ & 0,5935 & $12^{\circ}$ & 0,4951 & $6^{\circ}$ & 0,5475 \\
\hline Sicoob Caçador SC & $24^{\circ}$ & $13^{\circ}$ & 0,5659 & $11^{\circ}$ & 0,4987 & $23^{\circ}$ & 0,4460 \\
\hline
\end{tabular}

Nota. Fonte: Dados da pesquisa.

A partir da Tabela 1, é possível perceber o ranking de eficiências das cooperativas analisadas em cada um dos períodos. Cabe destacar que o TOPSIS constrói o ranking considerando os maiores e os menores scores apresentados, ou seja, leva em consideração a distância de ambos os lados para a determinação dos scores.

Assim, se uma determinada cooperativa apresentar o melhor índice em todos os indicadores analisados, ela obterá um score igual a 1,00, e se o índice for o pior em todos os indicadores analisados, seu score seria igual a 0,00. Neste sentido, percebese que não houve nenhuma cooperativa que apresentou melhor desempenho em todos os indicadores, assim como também não houve nenhuma cooperativa que tenha 
Análise da Eficiência nas Taxas de Retorno e Custos de Cooperativas de Crédito Brasileiras: uma Aplicação do Método Pearls

Cassia Bellei, Vanderlei Gollo, Juliana Fabris, Naline Tres

apresentado o pior desempenho em todos os indicadores, em nenhum dos períodos analisados.

As cooperativas estão apresentadas por ordem de tamanho, da maior para a menor, tomando como base os critérios do BACEN. Nota-se que a eficiência das cooperativas, em termos de taxas de retorno e custos, não tem relação com o tamanho, visto que a cooperativa mais eficiente em 2015 (Crediseara) encontra-se na $17^{\text {a }}$ posição em relação ao seu tamanho, apresentando score de eficiência de 0,6425. Em 2016, a cooperativa mais eficiente foi a Unicredi Oeste e Seara com score de 0,7522, ocupando a $6^{a}$ posição em relação ao tamanho. Já para 2017, a mais eficiente entre as cooperativas analisadas é a Sicoob São Miguel SC, com score de 0,6067 e $2^{\underline{a}}$ no ranking do BACEN.

Entre as menos eficientes para os períodos analisados estão a Cresol Concórdia para o período de 2015, Sulcredi Crediluz em 2016 e Sicoob Oestecredi em 2017. Mesmo as menos eficientes ficaram todas com índices próximos a 0,40 , sendo que este índice indica a distância para o pior cenário.

Corroborando com Gollo e Da Silva (2015), pode-se afirmar que a eficiência das cooperativas de crédito está relacionada principalmente à sua política de atuação, visto que as cooperativas têm liberdade para determinar taxas de juros e remuneração sobre investimentos, afetando diretamente o seu desempenho. Como nenhuma cooperativa apresentou índices próximos a 1,00 e também não se aproximaram de 0,00 (zero), pode-se afirmar que elas apresentam desempenho semelhante entre os indicadores analisados e, consequentemente, níveis de eficiência também semelhantes.

A maior oscilação entre a cooperativa mais e menos eficiente é percebida em 2016, em que a Unicredi Oeste e Seara se destacou em relação às demais e aos demais períodos. Esse fato se deve ao seu melhor desempenho em um conjunto maior de indicadores em relação às demais cooperativas, ou seja, foi o período em que uma cooperativa mais se aproximou do ideal entre as analisadas.

A fim de demonstrar a diferença de um ranking simples para o Ranking do Topsis, foi elaborado um ranking de pontuação a partir do desempenho das 
Análise da Eficiência nas Taxas de Retorno e Custos de Cooperativas de Crédito Brasileiras: uma

Aplicação do Método Pearls

Cassia Bellei, Vanderlei Gollo, Juliana Fabris, Naline Tres

cooperativas em cada um dos períodos analisados, conforme pode ser observado na Tabela 2.

Tabela 2

Ranking de eficiência a partir de pontuação simples

\begin{tabular}{|c|c|c|c|c|c|c|c|c|}
\hline COOPERATIVAS & $\begin{array}{r}2 \\
\text { Topsis }\end{array}$ & $\begin{array}{l}5 \\
\text { Pontos }\end{array}$ & $\begin{array}{r}2 \\
\text { Topsis }\end{array}$ & $\begin{array}{l}2016 \\
\text { s Pontos }\end{array}$ & $\begin{array}{r}2 \\
\text { s Topsis }\end{array}$ & $\begin{array}{l}2017 \\
\text { s Pontos }\end{array}$ & $\begin{array}{c}\text { Pontuação } \\
\text { Total }\end{array}$ & Ranking \\
\hline Cresol Xaxim & $3^{\circ}$ & 22 & $2^{\circ}$ & 23 & $4^{\circ}$ & 21 & 66 & $1^{0} \underline{0}$ \\
\hline Cresol Xavantina & $2^{o}$ & 23 & $5^{0}$ & 20 & $3^{\circ}$ & 22 & 65 & $2^{o}$ \\
\hline Sicoob Credirio SC & $4^{\circ}$ & 21 & $3^{\circ}$ & 22 & $11^{\circ}$ & 14 & 57 & $3^{\circ}$ \\
\hline Sicoob Creditapiranga SC/RS & $7^{0}$ & 18 & $9^{\circ}$ & 16 & 5oㅇ & 20 & 54 & $4^{0}$ \\
\hline Crediseara & $1^{0}$ & 24 & $7^{0} \underline{0}$ & 18 & $13^{0}$ & 12 & 54 & $4^{0}$ \\
\hline Sicoob São Miguel SC & $5^{0}$ & 20 & $18^{\circ}$ & 7 & $1 \stackrel{0}{\circ}$ & 24 & 51 & $6^{\circ}$ \\
\hline Cresol Chapecó & $6^{\circ}$ & 19 & $12^{0}$ & 13 & $6^{\circ}$ & 19 & 51 & $6^{\circ}$ \\
\hline Sicoob Credial SC & $8^{0}$ & 17 & $10^{\circ}$ & 15 & $10^{\circ}$ & 15 & 47 & $8^{\circ}$ \\
\hline Unicredi Oeste e Seara & $20^{\circ}$ & 5 & $1^{\circ}$ & 24 & $8^{\circ}$ & 17 & 46 & $9^{\circ}$ \\
\hline Sicoob Maxicrédito & $9^{\circ}$ & 16 & $4^{0}$ & 21 & $21^{\circ}$ & 4 & 41 & $10^{\circ}$ \\
\hline Sicoob Noroeste SC & $17^{\circ}$ & 8 & $19^{\circ}$ & 6 & $2^{\circ}$ & 23 & 37 & $11^{\circ}$ \\
\hline Sicoob Crediauc SC & $11^{\circ}$ & 14 & $16^{\circ}$ & 9 & $12^{0}$ & 13 & 36 & $12^{0}$ \\
\hline Sicoob Transcredi & $12^{0}$ & 13 & $13^{\circ}$ & 12 & $14^{0}$ & 11 & 36 & $12^{0}$ \\
\hline Sicoob Crediplanalto SC/RS & $15^{\circ}$ & 10 & $8^{\circ}$ & 17 & $19^{\circ}$ & 6 & 33 & $14^{\circ}$ \\
\hline Sicoob Credimoc SC & $16^{\circ}$ & 9 & $22^{\circ}$ & 3 & $7^{0}$ & 18 & 30 & $15^{\circ}$ \\
\hline Sicoob Videira SC & $18^{\circ}$ & 7 & $6^{0}$ & 19 & $22^{0}$ & 3 & 29 & $16^{\circ}$ \\
\hline Sicoob Caçador SC & $13^{\circ}$ & 12 & $11^{\circ}$ & 14 & $23^{\circ}$ & 2 & 28 & $17^{\circ}$ \\
\hline Sicoob Creditaipu & $14^{\circ}$ & 11 & $17^{\circ}$ & 8 & $18^{\circ}$ & 7 & 26 & $18^{\circ}$ \\
\hline Sulcredi Ouro & $22^{\circ}$ & 3 & $14^{\circ}$ & 11 & $15^{0}$ & 10 & 24 & $19^{\circ}$ \\
\hline Cresol Pedra Branca & $23^{\circ}$ & 2 & $21^{\circ}$ & 4 & 90 & 16 & 22 & $20^{\circ}$ \\
\hline Sicoob Oestecredi & $10^{\circ}$ & 15 & $20^{\circ}$ & 5 & $24^{\circ}$ & 1 & 21 & $21^{\circ}$ \\
\hline Cresol Concórdia & $24^{\circ}$ & 1 & $15^{0}$ & 10 & $16^{\circ}$ & 9 & 20 & $22^{\circ}$ \\
\hline Sicoob Valcredi Sul & $18^{\circ}$ & 7 & $23^{\circ}$ & 2 & $20^{\circ}$ & 5 & 14 & $23^{\circ}$ \\
\hline Sulcredi Crediluz & $21^{\circ}$ & 4 & $24^{\circ}$ & 1 & $17^{0}$ & 8 & 13 & $24^{\circ}$ \\
\hline
\end{tabular}

Nota. Fonte: Dados da pesquisa.

Para construir o ranking de eficiência, adotou-se atribuição de pontuação de acordo com a posição atingida pela cooperativa em cada um dos períodos. Assim, atribuiu-se o total de 24 pontos para a cooperativa mais eficiente, 23 pontos para a segunda mais eficiente e assim sucessivamente. A pontuação total é formada pela soma simples da pontuação atingida em cada um dos períodos analisados.

Com esta pontuação estabelecida, uma cooperativa poderia chegar a 72 pontos caso atingisse o melhor índice de eficiência em todos os períodos e 3 pontos se o seu índice de eficiência fosse o menor nos três períodos. 
Análise da Eficiência nas Taxas de Retorno e Custos de Cooperativas de Crédito Brasileiras: uma Aplicação do Método Pearls Cassia Bellei, Vanderlei Gollo, Juliana Fabris, Naline Tres

É possível verificar, a partir da Tabela 2, que, somando-se os 3 anos de score, a cooperativa Cresol Xaxim atingiu o primeiro lugar com 66 pontos. Na quarta posição houve um empate entre as cooperativas Creditapiranga SC/RS e a Crediseara, ambas com 54 pontos. Também houve empate na sexta posição, com 51 pontos (Sicoob São Miguel SC e Cresol Chapecó) e na décima segunda posição, com 36 pontos (Sicoob Crediauc SC e Sicoob Transcredi).

Entre as menos eficientes estão a Sicoob Valcredi Sul, na penúltima posição, com 14 pontos, e Sicredi Crediluz, que ficou na última colocação no ranking, somando apenas 13 pontos. Vale destacar que esses resultados são válidos e se aplicam à amostra analisada; portanto, qualquer cooperativa que seja incluída ou excluída da amostra poderá alterar os índices de eficiência, bem como o ranking de pontuação.

Este tipo de ranking por pontuação apresenta a posição por ordem de classificação, sem demonstrar proximidade ou distância no índice de eficiência, fator este que pode ser observado pela aplicação do Topsis, conforme apresentado na Tabela 3. 
Análise da Eficiência nas Taxas de Retorno e Custos de Cooperativas de Crédito Brasileiras: uma Aplicação do Método Pearls Cassia Bellei, Vanderlei Gollo, Juliana Fabris, Naline Tres

Tabela 3

Eficiência global das cooperativas pela aplicação do Topsis

\begin{tabular}{|c|c|c|c|}
\hline \multirow{2}{*}{ Cooperativas } & \multirow{2}{*}{ Ranking BACEN } & \multicolumn{2}{|c|}{ TOPSIS } \\
\hline & & Score & Posição \\
\hline Unicredi Oeste e Seara & $6^{0}$ & 0,6634 & $1^{0}$ \\
\hline Cresol Xaxim & 190 & 0,6096 & 20 \\
\hline Cresol Xavantina & $18^{\circ}$ & 0,5903 & $3^{\circ}$ \\
\hline Crediseara & $17^{\circ}$ & 0,5632 & $4^{\circ}$ \\
\hline Sicoob São Miquel SC & $2^{\circ}$ & 0,5549 & $5^{\circ}$ \\
\hline Sicoob Credirio SC & $13^{\circ}$ & 0,5517 & $6^{\circ}$ \\
\hline Sicoob Creditapiranga SC/RS & $10^{\circ}$ & 0,5139 & $7^{\circ}$ \\
\hline Cresol Chapecó & $23^{\circ}$ & 0,5004 & $8^{\circ}$ \\
\hline Sicoob Noroeste SC & $15^{\circ}$ & 0,4950 & 9은 \\
\hline Sicoob Credial SC & $5^{\circ}$ & 0,4628 & $10^{\circ}$ \\
\hline Sicoob Maxicrédito & 10 & 0,4156 & $11^{\circ}$ \\
\hline Sicoob Crediauc SC & $3^{\circ}$ & 0,4139 & $12^{\circ}$ \\
\hline Sicoob Transcredi & 90 & 0,3992 & $13^{0}$ \\
\hline Sicoob Credimoc SC & $14^{\circ}$ & 0,3843 & $14^{\circ}$ \\
\hline Sicoob Crediplanalto SC/RS & $12^{\circ}$ & 0,3819 & $15^{\circ}$ \\
\hline Sicoob Creditaipu & $4^{\circ}$ & 0,3635 & $16^{\circ}$ \\
\hline Sulcredi Ouro & $20^{\circ}$ & 0,3515 & $17^{\circ}$ \\
\hline Sicoob Videira SC & 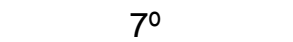 & 0,3510 & $18^{\circ}$ \\
\hline Cresol Pedra Branca & $22^{\circ}$ & 0,3506 & 190 \\
\hline Sicoob Cacador SC & $24^{\circ}$ & 0,3190 & $20^{\circ}$ \\
\hline Sicoob Oestecredi & $8^{\circ}$ & 0,2902 & $21^{\circ}$ \\
\hline Cresol Concórdia & $21^{\circ}$ & 0,2835 & $22^{\circ}$ \\
\hline Sicoob Valcredi Sul & $11^{\circ}$ & 0,2787 & $23^{\circ}$ \\
\hline Sulcredi Crediluz & $16^{\circ}$ & 0,2727 & $24^{\circ}$ \\
\hline
\end{tabular}

Nota. Fonte: Dados da pesquisa.

Com o objetivo de apresentar um ranking de eficiência demonstrado por meio de um índice, foi calculado novo Topsis a partir dos índices de eficiência apresentados pelas cooperativas analisadas, considerando-se os três períodos. Neste caso, também os índices poderiam variar de 1,00 a 0,00 (zero), em função do desempenho apresentado por cada uma das cooperativas.

Como nenhuma cooperativa foi a mais eficiente nos três períodos, não houve índice 1,00. A cooperativa mais eficiente em taxas de retorno e custos para os três períodos analisados foi a Unicredi Oeste e Seara com índice de eficiência de 0,6634. Isso significa que obteve maior proximidade dos melhores desempenhos atingidos pelas cooperativas da amostra em cada um dos períodos, conforme pode ser observado na Tabela 3. 
$\mathrm{Na}$ segunda colocação, encontra-se a Cresol Xaxim, com índice de 0,6096, seguida pela Cresol Xavantina, que apresentou índice de eficiência de 0,5903. Com a utilização do Topsis, é possível avaliar a distância entre as cooperativas no ranking final. Assim, nota-se uma distância maior entre a primeira e segunda colocada $(0,6634-$ $0,6096)$ do que entre a segunda e a terceira $(0,6096-0,5903)$, demonstrando que estas obtiveram um desempenho mais parecido em relação à primeira comparação.

Pode-se observar também um desempenho semelhante entre as cooperativas Sicoob São Miguel SC e Sicoob Credirio SC, com índices de 0,5549 e 0,5517, respectivamente, classificando-as na quinta e sexta colocação no ranking. As cooperativas Sicoob Maxicrédito e Sicoob Crediauc SC, com índices de 0,4156 e 0,4139, respectivamente, estão na décima e décima primeira posições no ranking de eficiência, sendo que, em termos de tamanho, representam a maior e terceira maior cooperativa da amostra, respectivamente. Isso demostra que a eficiência em custos e retornos não está necessariamente relacionada ao tamanho da cooperativa.

Pode-se afirmar ainda que, apesar de figurarem na parte baixa do ranking de desempenho, as cooperativas Sulcredi Ouro, Sicoob Videira SC e Cresol Pedra Branca apresentaram desempenho bastante semelhante, com índices de 0,3515, 0,3510 e 0,3506 respectivamente. Isso indica que possuem políticas de taxas e remuneração de investimentos bastante parecidos, em função dos resultados apresentados.

Entre as cooperativas menos eficientes da amostra, encontram-se a Sicoob Valcredi Sul e Sulcredi Crediluz, que figuram na vigésima terceira e vigésima quarta posição, com índices de 0,2787 e 0,2727 respectivamente. Os índices demonstram que estas cooperativas obtiveram um desempenho mais distante do considerado ideal pelo modelo. 
Análise da Eficiência nas Taxas de Retorno e Custos de Cooperativas de Crédito Brasileiras: uma Aplicação do Método Pearls Cassia Bellei, Vanderlei Gollo, Juliana Fabris, Naline Tres

\section{CONSIDERAÇÕES FINAIS}

As cooperativas de crédito necessitam medir esforços com o intuito de maximizar os resultados e atingir maiores padrões de eficiência (Bressan et al., 2011). Quanto maior a capacidade de maximização no atendimento às necessidades dos cooperados, por meio de produtos e serviços, manifesta-se de forma significativa a melhora na estrutura, desempenho e eficiência.

Com o objetivo de analisar o índice de eficiência no desempenho de cooperativas de crédito a partir da aplicação PEARLS, grupo $R$ taxas de retorno e custos, foi realizada pesquisa descritiva, conduzida por meio de análise documental, abordagem quantitativa e com o emprego do método TOPSIS para determinar um score a partir do melhor e do pior cenário, para cada cooperativa.

Os resultados obtidos indicam o nível de eficiência de cada cooperativa em relação às demais cooperativas que compõem a amostra; no entanto, o modelo não separa as cooperativas em eficientes e não eficientes, apenas determina um score de eficiência para cada uma delas, a partir dos indicadores apresentados.

Foi possível constatar que o índice de eficiência nas cooperativas analisadas não está relacionado ao montante de ativos que a cooperativa possui e, sim, às taxas de juros e retornos que as cooperativas praticam. Tal fato pode ser comprovado à medida que as maiores ou menores cooperativas da amostra não apresentaram índice de eficiência que mantivesse sua classificação na mesma posição de seu tamanho. Ou seja, pode-se verificar que algumas cooperativas menores apresentaram-se mais eficientes que outras maiores.

Os resultados obtidos por meio da pesquisa corroboram o estudo de Bressan et al. (2014), os quais ratificaram que os indicadores do sistema PEARLS são relevantes para análises relativas à insolvência, assim como análises financeiras e de risco tanto para cooperativas de crédito singulares quanto para as cooperativas centrais de crédito.

Como limitação da pesquisa, destaca-se que, apesar de os resultados do estudo serem válidos para a amostra analisada, não podem ser generalizados, pois, a partir do 
momento que se altera o tamanho da amostra, é provável que os resultados possam sofrer variações. No entanto, por meio dos resultados encontrados, reiterou-se a importância das informações contábeis para a análise das cooperativas de crédito, uma vez que os desempenhos obtidos sinalizaram que a maior parte das cooperativas da amostra está operando com eficiência no período analisado.

Por fim, espera-se que o presente estudo colabore com gestores e estudiosos na avaliação da eficiência no desempenho, em termos de taxas de retorno e custos, das cooperativas de crédito singulares, servindo como base comparativa para outros estudos. No mesmo sentido, sugere-se que pesquisas futuras possam contribuir e ampliar as discussões a partir de novos estudos, ou mesmo, da replicação deste para outros contextos, com ampliação da amostra, bem como agregando indicadores qualitativos que possam enriquecer as análises em bases comparativas.

\section{REFERÊNCIAS}

Araújo, E. A. T., \& Silva, W. A. C. (2011). Cooperativas de crédito: a evolução dos principais sistemas brasileiros com um enfoque em indicadores econômicofinanceiros. Contextus-Revista Contemporânea de Economia e Gestão, 9(1), 117126.

Banco Central do Brasil [BACEN]. O que é cooperativa de crédito? Recuperado de: $<$ https://www.bcb.gov.br/estabilidadefinanceira/cooperativacredito>, Acesso em: 27/nov./2019.

Bressan, V. G. F., Braga, M. J., Bressan, A. A., \& Resende, M. A., Fo. (2011). Uma aplicação do sistema PEARLS às cooperativas de crédito brasileiras. Revista de Administração, 46(3), 258-274.

Bressan, V. G. F., Bressan, A. A., Oliveira, P. H. M., \& Braga, M. J. (2014). Quais indicadores contábeis financeiros do Sistema PEARLS são relevantes para análise de insolvência das cooperativas centrais de crédito no Brasil? Contabilidade Vista \& Revista, 25(1), 74-98.

Bulgurcu, B. K. (2012). Application of TOPSIS technique for financial performance evaluation of technology firms in Istanbul stock exchange market. Procedia-Social and Behavioral Sciences, 62, 1033-1040. 
Camargos, M. A., \& Barbosa, F. V. (2005). Análise do desempenho econômicofinanceiro e da criação de sinergias em processos de fusões e aquisições do mercado brasileiro ocorridas entre 1995 e 1999. Caderno de Pesquisas em Administração, 12(2), 99-115.

Chaves, S. S. (2011). O cooperativismo de crédito no Brasil: evolução e perspectivas. In: Dodl, A., \& Barros, J. (Org.). Desafios do sistema financeiro nacional: o que falta para colher os benefícios da estabilidade conquistada. (69-97). Rio de Janeiro: Elsevier-Campus.

Cunha, P. R., Santos, V., Bezerra, F. A., \& Pinto, V. M. (2010). Custos Políticos nas Instituições Financeiras face à resolução 3.518/07 do Bacen. Revista Base (Administração e Contabilidade) da UNISINOS, 7(2), 94-107.

Silva, A., Padilha, E. S., \& Silva, T. P. (2015). Análise da performance econômicofinanceira das 25 maiores cooperativas de crédito brasileiras. Desenvolvimento em Questão, 13(32), 303-333.

Gil, A. C. (2019). Métodos e técnicas de pesquisa social. (7a ed). São Paulo: Atlas.

Gollo, V., \& da Silva, T. P. (2015). Eficiência no desempenho econômico-financeiro de cooperativas de crédito brasileiras. Revista de Contabilidade $e$ Organizações, 9(25), 43-55.

Kaplan, R. S. \& Cooper, R. (2000). Custo e Desempenho: administre seus custos para ser competitivo. São Paulo: Futura.

Lima, M. D. S. M. C., Silva, B. S., \& Lima, C. C. (2013). A Importância do cooperativismo de crédito no desenvolvimento regional. Revista Opara, 3(1).

Maia, S. C., Bressan, V. G. F., Lamounier, W. M., \& Braga, M. J. (2013). Gerenciamento de resultados em cooperativas de crédito no Brasil. Brazilian Business Review, 10(4), 96-116.

McKillop, D. G., Glass, J. C., \& Ferguson, C. (2002). Investigating the cost performance of UK credit unions using radial and non-radial efficiency measures. Journal of Banking \& Finance, 26(8), 1563-1591.

Menezes, C. M., \& Lajús, M. (2015). Cooperativismo de crédito e desenvolvimento. Revista Economia e Desenvolvimento, 14(2), 294-313. 
Oliveira, P. H. M., Bressan, V. G. F., \& Bressan, A. A. (2014). Existe diferença no desempenho financeiro das cooperativas centrais de crédito no Brasil? Revista Evidenciação Contábil \& Finanças, 2(2), 40-54.

Pinheiro, M. A. H. (2008). Cooperativas de crédito: história da evolução normativa no Brasil. 6. ed. Brasília, BCB. Recuperado de $<$ https://www.bcb.gov.br/htms/public/microcredito/livro_cooperativas_credito.pdf?idi oma $=\mathrm{P}>$. Acesso em: 27/nov./2019.

Raupp, F. M. \& Beuren, I. M. (2012). Metodologia da pesquisa aplicável às ciências sociais. In: Beuren, I. M. (Org.). Como elaborar trabalhos monográficos em contabilidade: teoria e prática. (3a ed)., 76-97. São Paulo: Atlas.

Richardson, D. C. (2002). PEARLS monitoring system. World Council of Credit Unions, Madison. 4. Oct. Recuperado de <http://www.coopdevelopmentcenter.coop>. Acesso em: 20/fev./2019.

Soares, M. M., \& Melo, A. D., Sobo. (2008). O papel do Banco Central do Brasil e a importância do cooperativismo de crédito. (2a ed). Brasília: BCB.

World Council of Credit Unions [WOCCU]. (2014). Statistical Report 2014. Washington, World Council of Credit Unions. Recuperado de: <http://www.woccu.org/our_network/statreport>. Acesso em: 09/ago./2019.

World Council of Credit Unions [WOCCU]. (2018). Annual Report 2018. Washington, World Council of Credit Unions. Recuperado de: <http://www.woccu.org/documents/2018WorldCouncilAnnualReport>. Acesso em: 09/ago./2019.

${ }^{*}$ Nota:

O presente trabalho foi realizado com apoio da Coordenação de Aperfeiçoamento de Pessoal de Nível Superior - Brasil (CAPES) - Código de Financiamento 001.

Data de Submissão: 29/11/2019

Data de Aceite: 17/12/2019 